\title{
Electronic Health Record-Based Patient Identification and Individualized Mailed Outreach for Primary Cardiovascular Disease Prevention: A Cluster Randomized Trial
}

\author{
Stephen D. Persell, MD, MPH', ${ }^{1,2}$ Donald M. Lloyd-Jones, MD, SCM ${ }^{3,4}$, Elisha M. Friesema, BA ${ }^{7}$, \\ Andrew J. Cooper, $\mathrm{MPH}^{7}$, and David W. Baker, $\mathrm{MD}, \mathrm{MPH}^{1,2}$
}

'Division of General Internal Medicine and Geriatrics, Feinberg School of Medicine, Northwestern University, Chicago, IL, USA; ${ }^{2}$ Institute for Health Care Studies, Feinberg School of Medicine, Northwestern University, Chicago, IL, USA; ${ }^{3}$ Division of Cardiology, Feinberg School of Medicine, Northwestern University, Chicago, IL, USA; ${ }^{4}$ Department of Preventive Medicine, Feinberg School of Medicine, Northwestern University, Chicago, IL, USA.

BACKGROUND: Many individuals at higher risk for cardiovascular disease (CVD) do not receive recommended treatments. Prior interventions using personalized risk information to promote prevention did not test clinic-wide effectiveness.

OBJECTIVE AND DESIGN: To perform a 9-month cluster-randomized trial, comparing a strategy of electronic health record-based identification of patients with increased CVD risk and individualized mailed outreach to usual care.

PARTICIPANTS: Patients of participating physicians with a Framingham Risk Score of at least $5 \%$, lowdensity lipoprotein (LDL)-cholesterol level above guideline threshold for drug treatment, and not prescribed a lipid-lowering medication were included in the intention-to-treat analysis.

INTERVENTION: Patients of physicians randomized to the intervention group were mailed individualized CVD risk messages that described benefits of using a statin (and controlling hypertension or quitting smoking when relevant).

MAIN MEASURES: The primary outcome was occurrence of a LDL-cholesterol level, repeated in routine practice, that was at least $30 \mathrm{mg} / \mathrm{dl}$ lower than prior. A secondary outcome was lipid-lowering drug prescribing. Clinicaltrials.gov identifier: NCT01286311.

KEY RESULTS: Fourteen physicians with 218 patients were randomized to intervention, and 15 physicians with 217 patients to control. The mean patient age was 60.7 years and $77 \%$ were male. There was no difference in the primary outcome (11.0 \% vs. $11.1 \%$, OR 0.99, $95 \%$ CI 0.56-1.74, $P=0.96)$, but intervention group patients were twice as likely to receive a prescription for lipid-lowering medication (11.9\%, vs. $6.0 \%$, OR 2.13, $95 \%$ CI 1.05-4.32,

Electronic supplementary material The online version of this article (doi:10.1007/s11606-012-2268-1) contains supplementary material, which is available to authorized users.

Grant support: K08 HS015647, Agency for Healthcare Research and Quality Trial registration: Clinicaltrials.gov identifier: NCTO1286311.

Received May 23, 2012

Revised October 12, 2012

Accepted October 17, 2012

Published online November 11, 2012 $p=0.038$ ). In post hoc analysis with extended follow-up to 18 months, the primary outcome occurred more often in the intervention group $(22.5 \%$ vs. $16.1 \%$, OR 1.59, $95 \%$ CI 1.05-2.41, P=0.029).

CONCLUSIONS: In this effectiveness trial, individualized mailed CVD risk messages increased the frequency of new lipid-lowering drug prescriptions, but we observed no difference in proportions lowering LDL-cholesterol after 9 months. With longer follow-up, the intervention's effect on LDL-cholesterol levels was apparent.

KEY WORDS: cholesterol; primary care; cardiovascular disease prevention; electronic health records; patient outreach.

$\mathrm{J}$ Gen Intern Med 28(4):554-60

DOI: $10.1007 /$ s11606-012-2268-1

(C) Society of General Internal Medicine 2012

\section{BACKGROUND}

Cardiovascular disease (CVD) risk assessment is an important step in determining who will benefit from preventive treatments like the use of statins. Guidelines ${ }^{1-3}$ and performance measures ${ }^{4}$ that address primary CVD prevention recommend using quantitative risk assessment. However, risk assessment is not often performed in primary care, and physicians may have inaccurate perceptions of patients' risk. ${ }^{5,6}$ As a result, some patients whose risk is high enough to warrant intervention may go unrecognized. There is some evidence that primary prevention approaches that use calculation of CVD risk lead to increased treatment of risk factors by physicians, and can increase patients' intent to start therapies, such as lipid-lowering medication or aspirin, by about 15 to $30 \% .^{7-13}$ Data contained in an electronic health record (EHR) can be used to identify candidates for risk-reducing interventions. ${ }^{14,15}$ Automated identification of these patients makes possible practice-wide quality improvement activities delivered directly to patients, such as the approach we tested here. We hypothesized that patientdirected, individualized risk messages delivered outside of 
routine office care would result in improved treatment and control of low-density lipoprotein (LDL) cholesterol.

\section{METHODS}

\section{Objectives and Design}

We performed a pragmatic clinical trial, ${ }^{16}$ to test the effectiveness of a clinical care strategy that included EHRbased identification of patients with increased CVD risk and mailed outreach with individualized CVD risk information delivered at the level of a physicians' practice. To do this, we performed a 9-month cluster randomized effectiveness study, with clusters determined at the level of the primary care physician. The study took place between February 3rd and November 3rd, 2011.

\section{Participants}

Northwestern University's Institutional Review Board (IRB) approved the study. Internal medicine primary care physicians at a large practice affiliated with an academic medical center and their patients were eligible for inclusion. Characteristics of all patients seen at the practice during 2011 are provided in the Appendix available on line. The practice had used the same EHR for over 13 years. Physicians routinely received reports about quality of care measures. Patients rarely were sent mailings other than for test results.

Physicians provided written informed consent to be randomized, and to have study staff mail risk messages directly to patients on their behalf if they were randomized to the intervention. Patients were included with a waiver of consent.

Patients who met the following eligibility criteria were included. Their primary care physician was enrolled in the study, age was 40 to 79 years, the EHR medication list did not include a lipid-lowering medication, there was no diagnosis of coronary heart disease, heart failure, stroke, diabetes mellitus, or peripheral arterial disease in the EHR, and two or more visits to any provider in the clinic occurred in the preceding 24 months. Patients also had to have a LDL cholesterol test performed in the past 5 years; in addition, their most recent LDL cholesterol was $\geq 100 \mathrm{mg} /$ $\mathrm{dl}$ and the Framingham Risk Score (FRS) — risk of nonfatal myocardial infarction or coronary death during the next 10 years ${ }^{17}$ — was $>20 \%$, LDL cholesterol was $\geq 130 \mathrm{mg} / \mathrm{dl}$ and the FRS was 10 to $20 \%$, or LDL cholesterol was $\geq$ $160 \mathrm{mg} / \mathrm{dl}$ and the FRS was 5 to $<10 \%$. The FRS was calculated from EHR data retrieved through an electronic query of Northwestern University's enterprise data warehouse. We had previously shown that the FRS could be reliably determined from searchable EHR data. ${ }^{15}$ FRS was calculated using the continuous equations employed by the
National Heart, Lung and Blood's National Cholesterol Education Program's online risk calculator. ${ }^{17}$ This study did not include patients with diabetes or established CVD, because ongoing quality improvement activity already addressed these conditions. ${ }^{18}$

\section{Intervention}

The intervention took a population approach to uncontrolled cholesterol in a primary care. We used EHR data to identify the target population, and alerted intervention physicians to their patients who were not meeting guideline goals for LDL cholesterol control based on their CVD risk level, and who were not treated with a lipid-lowering drug. Intervention development was informed by theoretical work on health behavior change, such as the Integrated Behavioral Model. ${ }^{19}$ The written risk information was designed to: 1 ) increase a patient's sense of susceptibility to CVD; 2) provide normative information from a trusted source (the primary care doctor) about health behaviors (e.g. taking medication) that a patient can adopt to lower his or her risk; and 3) promote the notion that taking action to lower risk is feasible. Content was presented to two focus groups comprised of nine primary care physicians. The groups reviewed the content and intervention procedures and provided feedback. Messages were pilot-tested with several non-clinician volunteers and one patient (using his actual risk information). Pilot testers identified areas requiring clarification. We incorporated feedback from focus groups and pilot tests into the final design.

Physicians randomized to the intervention received a secure email within the EHR with a list of their eligible patients, along with the FRS and Global Cardiovascular Risk Score. ${ }^{20}$ Physicians were also told when patients had uncontrolled hypertension or were smokers. A sample physician message is provided in the Appendix (available online). Physicians could indicate patients they did not wish to receive outreach. All other patients were mailed a message containing their personal CVD information, depicted in written and graphic format and estimating the risk reduction that could be obtained using a statin (see Online Appendix). For patients who had uncontrolled hypertension or were smokers, the benefits of modifying these risk factors were also shown. The mailing, addressed from their primary care physician, encouraged patients to discuss risk-lowering options with them. After 9 months, control group physicians were provided with lists of their eligible patients and their risk scores.

\section{Outcomes}

We measured outcomes from data collected through routine care. We collected data using automated searches of standardized portions of the EHR. The primary study 
outcome was the occurrence of a repeat LDL Cholesterol that was at least $30 \mathrm{mg} / \mathrm{dl}$ lower than the baseline LDL cholesterol. We chose this as the primary outcome, because a decline of this magnitude would likely indicate that either drug therapy was begun or substantial dietary changes were undertaken. Secondary outcomes included: whether a lipidlowering drug was prescribed; whether aspirin or another antiplatelet drug was prescribed (among the subgroup not prescribed this treatment at baseline); change in systolic and diastolic blood pressures, and the difference in the number of antihypertensive drug classes prescribed (among patients who had uncontrolled hypertension at baseline); and documentation of quitting smoking (among patients who were smokers at baseline). Other processes examined were the number of in-person office visits, telephone and email contacts during the 9-month observation period. All outcomes were assessed by applying the outcome criteria to patient data automatically collected from EHRs using automated searches. No human judgment was involved in outcome assessments.

Exploratory analyses examined the frequency and timing of LDL-cholesterol testing with respect to the timing of prescribing a new lipid-lowering drug. We also conducted additional post hoc analyses using data collected within the 18 months after the start of the intervention.

\section{Sample Size}

The size of the study was constrained by being limited to one large group practice. Although the proportion of patients achieving the primary outcome over a 9-month period was not known a priori, we anticipated it would be low $(\leq 5 \%)$. On clinical grounds, we decided that this intervention would be potentially worthwhile if it raised the primary outcome by $10 \%$. With an independent sample, 159 patients per group would provide $80 \%$ power to detect a $10 \%$ difference with an alpha error rate of $5 \%$. We anticipated 25 to 35 clusters with approximately 15 patients per cluster, but the intra-cluster correlation of the outcome was not known a priori. If the intra-cluster correlation for the outcome were 0.02 , the estimated design effect would be approximately 1.28 , and an approximate sample size of 203 patients per arm would be needed to provide the power above.

\section{Randomization}

All physicians were enrolled prior to randomization. Physicians with no eligible patients were excluded. To help balance the number of eligible patients in the intervention and control groups, physicians were placed in blocks of four by number of eligible patients. Randomization was performed using a random number generator (SAS 9.2, SAS
Institute Inc., Cary, NC) by a researcher who was not aware of the physicians' order in the blocks. Allocation to intervention or control groups was not revealed until after randomization was completed. To prevent the two participating physicians with the largest number of eligible patients from being assigned to the same group, the first block of four were grouped in two groups of two (first and fourth largest comprised one group and second and third largest another), and these two groups were randomly assigned.

\section{Statistical Analysis}

Baseline characteristics of intervention and control group patients and physicians were compared using simple descriptive statistics as appropriate. All eligible patients of enrolled physicians were included in the analyses of binary outcomes according to the intention-to-treat principle. Because physicians rather than patients were randomized, we used generalized linear models with intervention group as the fixed effect and physician as random effects (PROC GLIMMIX, SAS version 9.2, SAS Institute Inc., Cary, NC). We used the logit link for binary outcomes, log link for count outcomes and identity link for normally distributed continuous outcomes. Models of blood pressure outcomes included patients' baseline values. All tests of significance were two-tailed.

\section{RESULTS}

Recruitment. Flow of study participants is depicted in Figure 1. Thirty-one of 37 physicians enrolled. Two had no eligible patients. The remaining 29 physicians had 435 eligible patients ( 218 were randomized to the intervention arm and 217 to the control arm). Five physicians in the intervention group indicated that they did not want the mailed outreach message to be sent to $14(6.4 \%)$ patients.

Participant Characteristics. The mean age of eligible patients was 60.7 years and $77 \%$ were male. Physicians and patient characteristics were similar in both groups (Table 1). Baseline diastolic blood pressure was $2 \mathrm{mmHg}$ greater among controls.

Intention-to-Treat Analysis. The primary outcome at 9 months occurred for $11.0 \%$ of intervention group patients and $11.1 \%$ of controls (odds ratio [OR] 0.99, $95 \%$ confidence interval [CI] 0.56-1.74, $P=0.96$ ). At 9 months, $11.9 \%$ of the intervention group and $6.0 \%$ of controls had received a lipid-lowering medication prescription (OR 2.13 [CI] 1.22-3.72). Intra-cluster correlation coefficients were 0.029 for the primary outcome and 0.011 for the prescription outcome. There 


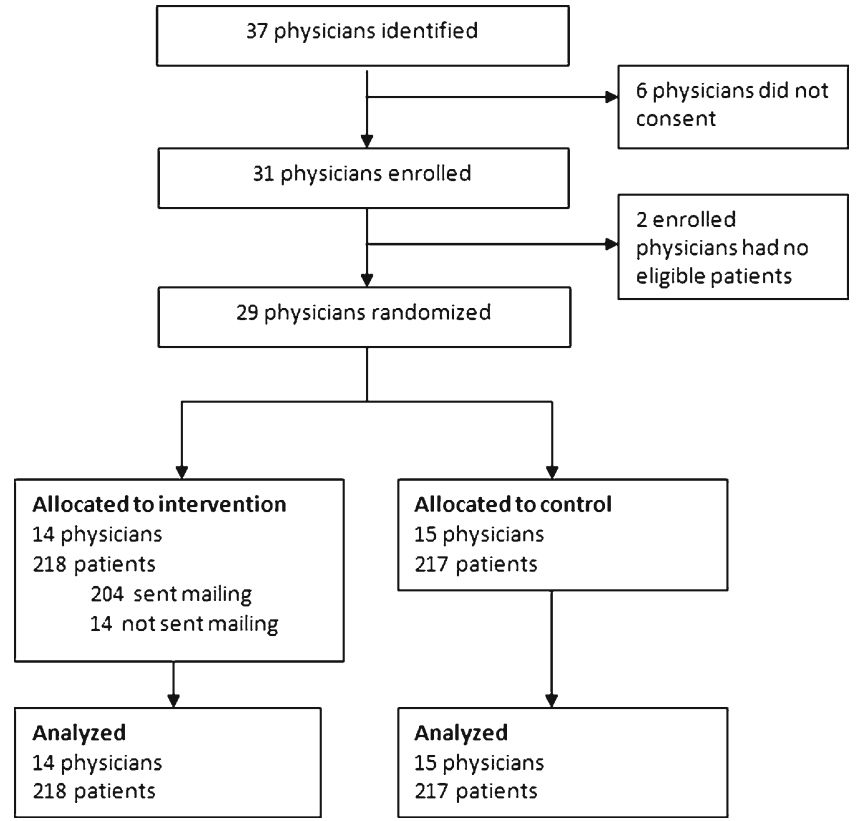

Figure 1. Flow diagram.

were no differences between groups for in-person visits, telephone calls or email contacts during the study (Table 2).

Table 1. Characteristics of Patients and Physicians

\begin{tabular}{|c|c|c|c|}
\hline Characteristic & Intervention & Control & $\mathbf{P}$ \\
\hline Patients & $N=218$ & $N=217$ & \\
\hline Male, \% & 77.5 & 77.0 & 0.89 \\
\hline Age, y (SD) & $61.3(9.4)$ & $60.1(9.2)$ & 0.20 \\
\hline Race/ethnicity,\% & & & 0.43 \\
\hline White & 52.8 & 48.4 & \\
\hline African-American & 16.1 & 14.3 & \\
\hline Asian & 0.9 & 1.8 & \\
\hline Hispanic & 4.6 & 3.7 & \\
\hline Other & 10.6 & 9.2 & \\
\hline Unknown & 15.1 & 22.6 & \\
\hline $\begin{array}{l}\text { Framingham risk } \\
\text { score, } \% \text { (SD) }\end{array}$ & $14.2(6.7)$ & $13.8(6.3)$ & 0.49 \\
\hline $\begin{array}{l}\text { Global cardiovascular } \\
\text { risk score, } \%(\mathrm{SD})^{\mathrm{a}}\end{array}$ & $24.4(11.3)$ & $23.9(10.6)$ & 0.64 \\
\hline Total cholesterol, mg/dl (SD) & $228.1(32.7)$ & $225.9(29.4)$ & 0.46 \\
\hline LDL cholesterol, mg/dl (SD) & $156.5(26.4)$ & $156.3(24.6)$ & 0.92 \\
\hline HDL cholesterol, mg/dl (SD) & $44.2(13.5)$ & $43.5(12.1)$ & 0.58 \\
\hline Triglycerides, mg/dl (SD) & $137.4(70.2)$ & $132.5(75.7)$ & 0.49 \\
\hline $\begin{array}{l}\text { Systolic blood } \\
\text { pressure, } \mathrm{mm} \text { Hg (SD) }\end{array}$ & $135.2(16.1)$ & $137.2(16.6)$ & 0.20 \\
\hline $\begin{array}{l}\text { Diastolic blood } \\
\text { pressure, mm } \mathrm{Hg}(\mathrm{SD})\end{array}$ & $80.8(10.1)$ & $82.8(9.1)$ & 0.03 \\
\hline Drug treated hypertension, $\%$ & 56.0 & 55.8 & 0.97 \\
\hline Current smoking, $\%$ & 14.7 & 20.3 & 0.12 \\
\hline Aspirin treated, \% & 17.4 & 23.0 & 0.15 \\
\hline $\begin{array}{l}\text { Previously prescribed } \\
\text { lipid-lowering drug, \% }\end{array}$ & 14.7 & 13.4 & 0.69 \\
\hline Physicians & $N=14$ & $N=15$ & \\
\hline Male, \% & 50 & 40 & 0.59 \\
\hline $\begin{array}{l}\text { Number of eligible } \\
\text { patients, median (IQR) }\end{array}$ & $9(5-20)$ & $6(4-21)$ & 0.88 \\
\hline
\end{tabular}

$H D L$ high-density lipoprotein; IQR interquartile range; LDL lowdensity lipoprotein; SD standard deviation

${ }^{a}$ The Global Cardiovascular Risk Score is described in reference 17
Table 2. Outcomes at 9 Months

\begin{tabular}{|c|c|c|c|c|}
\hline & Intervention & Control & OR and 95 & $\mathbf{P}$ \\
\hline & $N=\mathbf{2 1 8}$ & $N=\mathbf{2 1 7}$ & & \\
\hline Primary endpoint & & & & \\
\hline $\begin{array}{l}\text { LDL-C repeated } \\
\text { and } \leq 30 \mathrm{mg} / \mathrm{dl} \\
\text { lower than } \\
\text { baseline, } \%\end{array}$ & 11.0 & 11.1 & $\begin{array}{l}0.99 \\
(0.56-1.74)\end{array}$ & 0.96 \\
\hline Secondary endpoints & & & & \\
\hline $\begin{array}{l}\text { Lipid-lowering } \\
\text { medication } \\
\text { prescribed, \% }\end{array}$ & 11.9 & 6.0 & $\begin{array}{l}2.13 \\
(1.22-3.72)\end{array}$ & 0.008 \\
\hline $\begin{array}{l}\text { LDL-C } \\
\text { repeated, } \%\end{array}$ & 38.1 & 33.6 & $\begin{array}{l}1.22 \\
(0.81-1.86)\end{array}$ & 0.34 \\
\hline $\begin{array}{l}\text { Any office } \\
\text { visit during } \\
\text { follow-up, \% }\end{array}$ & 61.9 & 56.7 & $\begin{array}{l}1.19 \\
(0.87-1.63)\end{array}$ & 0.28 \\
\hline $\begin{array}{l}\text { Number of } \\
\text { office visits, } \\
\text { median (IQR) }\end{array}$ & $1(0-2)$ & $1(0-1)$ & & 0.14 \\
\hline $\begin{array}{l}\text { Number of } \\
\text { telephone } \\
\text { contacts, } \\
\text { median (IQR) }\end{array}$ & $0(0-2)$ & $0(0-2)$ & & 0.75 \\
\hline $\begin{array}{c}\text { Emails to office, } \\
\text { median (IQR) }\end{array}$ & $0(0-1)$ & $0(0-0)$ & & 0.96 \\
\hline
\end{tabular}

CI confidence interval; IQR interquartile range; $L D L-C$ low-density lipoprotein cholesterol; OR odds ratio

Prespecified Subgroups. Among the 161 patients with uncontrolled hypertension at baseline, there were no significant differences in the characteristics listed in Table 1. Patients in the intervention group were more than twice as likely to have an increase in the number of antihypertensive drug classes prescribed, but this was not statistically significant (Table 3). Repeat office blood pressures were obtained for $63 \%$. Systolic and diastolic blood pressures were nonsignificantly lower in the intervention group (adjusted mean difference $-4.7 /-1.9 \mathrm{mmHg}$ ) (Table 3). Among the 347 patients who did not did not have aspirin on their medication list at baseline, rates at 9 months were $3.9 \%$ and $1.8 \%$ in the intervention and control groups, respectively (OR 2.18, $95 \%$ CI $0.50-9.5, p=0.30$ ). Among smokers, documentation of non-smoking during follow-up was rare and did not vary by group.

Post Hoc Analyses. Few patients had LDL testing within 9 months $(38.1 \%$ intervention, $33.6 \%$ control, $P=0.34)$. We examined timing of new lipid-lowering drug prescription with respect to repeat lab testing. In the control group, 0 of $13(0 \%)$ of individuals with a new medication had a LDL-cholesterol test after the new prescription was written. In the intervention group, 7 of 26 with a new lipid-lowering medication prescribed had LDL-cholesterol done afterwards. Of these, 6 of 7 had at least a $30 \mathrm{mg} / \mathrm{dl}$ reduction in LDL-cholesterol.

After 18 months of follow-up, $56.4 \%$ of the intervention group and $47.5 \%$ of controls had a repeat LDL test. The primary outcome at 18 months occurred for $22.5 \%$ of intervention group patients and $16.1 \%$ of controls (OR 
Table 3. Blood Pressure Related Outcomes at 9 Months Among Patients With Uncontrolled Hypertension at Baseline

\begin{tabular}{|c|c|c|c|c|}
\hline & $\begin{array}{l}\text { Intervention } \\
N=76\end{array}$ & Control $N=85$ & OR (95\% CI) & $\mathbf{P}$ \\
\hline Antihypertensive drug classes, median (IQR) & $1(1-2)$ & $1(0-2)$ & & 0.45 \\
\hline Increase in number of antihypertensive drug classes, $\%$ & $11.8 \%$ & $4.7 \%$ & $\begin{array}{l}2.89(0.70-11.9) \\
\text { Adjusted mean difference }(95 \% \mathrm{CI})^{\mathrm{b}}\end{array}$ & 0.14 \\
\hline Systolic blood pressure, $\mathrm{mm} \mathrm{Hg}(\mathrm{SD})^{\mathrm{a}}$ & $137.2(16.8) 51 / 76$ & $142.5(16.7) 51 / 85$ & $-4.7(1.8$ to -11.3$)$ & 0.15 \\
\hline Diastolic blood pressure, $\mathrm{mm} \mathrm{Hg}(\mathrm{SD})^{\mathrm{a}}$ & $80.3(9.7) 51 / 76$ & $81.9(10.5) 51 / 85$ & $-1.9(1.6$ to -5.4$)$ & 0.28 \\
\hline
\end{tabular}

CI confidence interval; IOR interquartile range; OR odds ratio

${ }^{a}$ The number of patients with follow-up data available is indicated

${ }^{b}$ Adjusted mean difference represents the intervention effect derived from models that include baseline blood pressure and physician-level random effects

$1.59,95 \%$ CI $1.05-2.41, P=0.029) ; 17.4 \%$ of patients in the intervention group and $11.1 \%$ of controls had received a lipid-lowering medication prescription (OR 1.67, $95 \% \mathrm{CI}$ $0.83-3.38, P=0.15)$.

\section{DISCUSSION}

In this physician-randomized effectiveness study, mailed individualized CVD risk messages to patients with uncontrolled LDL cholesterol resulted in a doubling in the proportion of patients who received a new lipid-lowering drug prescription. Among the subgroup with uncontrolled hypertension and the subgroup not using aspirin at baseline, prescribing additional antihypertensive medication and prescribing aspirin, respectively, were more common in the intervention group. Blood pressure was lower in the intervention group. However, subgroups eligible for these interventions were small, and these results did not achieve statistical significance. This intervention showed no effect on the primary outcome at the prespecified study end point (having a repeat LDL-cholesterol level obtained within 9 months that was $\geq 30 \mathrm{mg} / \mathrm{dl}$ lower than baseline); but after 18 months, there was a significant effect of the intervention on this outcome.

Our primary outcome, which relied on lab testing during routine care, hindered our ability to detect differences in LDL cholesterol lowering, because most patients did not have a repeat LDL-cholesterol test during the study period. Unlike studies with patient-level enrollment and measurement of outcomes performed as part of the study design, ${ }^{10,11,13,21}$ we used a pragmatic design to test the effects of this intervention on the entire population of eligible patients cared for by study physicians. Physicians, but not patients, were enrolled, and outcomes were obtained from routine clinical care. Most patients who received a new prescription did not have a repeat LDL-cholesterol test performed during the study period, and many who had the test received it prior to starting treatment. If pharmacy dispensing information were routinely available, examining drug initiation and persistence would be valuable ways to explore the effects of this intervention.

Even though the physician-randomized design that does not include patient enrollment limits the outcomes that can be assessed, this design is an important way to study this kind of intervention for two reasons. First, it shows the effects for the entire clinical population to which this kind of intervention may be applied. Studies with patient-level enrollment may not be readily generalizable to overall patient populations. Second, the acts of enrollment and follow-up may exert a strong effect on the control groups, thereby diminishing the perceived benefits of interventions. One study of CVD risk messages showed a small favorable effect on LDL cholesterol, but this intervention may have yielded more dramatic findings had there not been such a large cholesterol reduction among the control group. ${ }^{10}$

Even though we eventually observed differences in cholesterol lowering, the intervention effect was small. In the intervention group, $83 \%$ did not receive prescription lipid-lowering therapy after 18 months. However, this small effect size should be viewed in the context of the low resources required. Data from the EHR were prepared into a mailed intervention by a non-clinician staff member. Other CVD primary prevention studies achieved larger effects but required greater resources, and many included multiple contacts with clinicians. ${ }^{8,10,11,21-24}$ If a measurable positive effect of a more limited intervention like ours can be accomplished at a sufficiently low cost, it may be a worthwhile approach to adopt. If the process of delivering these messages can be fully automated (such as using a patient portal connected to the electronic health record), this may reduce the cost of providing this service further.

One potential reason for the small effect was that patients had only a single exposure to the messages. Prior studies with repeated exposure to risk messages and counseling have shown the most favorable results. ${ }^{8,10,11,22,23}$ An additional study is needed to examine whether repeated delivery increases efficacy.

Another reason that more intervention patients did not achieve the LDL control goal may have been that patients did not think their risk was particularly high. The mean 10year risk for any CVD event was $24 \%$. A prior study in patients with diabetes showed that a decision aid for statin use made risk perception more accurate, but statin adherence was not changed. ${ }^{25}$ Having more than a three in four chance of not having a CVD event over 10 years may have made medication use not seem worthwhile. It is possible 
that placing more emphasis on CVD risk over a longer time horizon (i.e. lifetime risk) or making other changes to the framing of the risk could increase the impact.

It is possible that combining this approach with other physician-directed interventions, such as reminders, performance audit and feedback, or financial incentives, could produce additive effects. Computerized clinical decision support has been used successfully to promote guidelinebased treatment of dyslipidemia in primary care. ${ }^{26}$ Future studies should aim to determine the optimal combination of quality improvement techniques.

These findings should be viewed with several additional limitations in mind. This study was performed at a single site, and as a result we do not know how the findings would differ in other settings. Intervention group physicians could have interacted with control group physicians, leading to alterations in their behavior, but since this was predominantly a patient-directed intervention, it is unlikely that there was a large contamination effect. The study size was adequate to safely exclude the $10 \%$ increase in the primary outcome we sought to detect, but power was limited for outcomes that apply to the subgroups (uncontrolled hypertension, aspirin non-users, and current smokers). Lastly, the smoking outcome, which is based on patients' self-report at the time of an office visit, may not be reliable.

This study demonstrated that practice-wide delivery of individualized CVD risk messages derived from EHR data to moderately-high and high-risk patients is feasible, and resulted in a modest increase in lipid-lowering drug prescribing. Ultimately, however, the absolute effects were small and most patients remained untreated. More powerful approaches are needed to address the burden of uncontrolled risk factors among individuals at increased risk for CVD. Providing patients with repeated individualized cardiovascular risk assessments with treatment recommendations may prove to be more effective than a single exposure.

Acknowledgements: Author contribution: Dr. Persell had full access to the study data and takes responsibility for the integrity of the data and the accuracy of the data analysis.

Study concept and design: Persell, Lloyd-Jones, Baker

Acquisition of data: Persell, Cooper, Friesema

Analysis and interpretation of data: Persell, Cooper

Drafting of the manuscript: Persell

Critical revision of the manuscript for important intellectual content:

Lloyd-Jones, Friesema, Cooper, Baker

Statistical analysis: Persell

Obtained funding: Persell

Study supervision: Persell, Baker

Funding/Support: K08 HSO15647, Agency for Healthcare Research and Quality.

Role of the funder: the funding organization had no role in the design and conduct of the study; collection, management, analysis, and interpretation of the data; and preparation, review, or approval of the manuscript

This work was presented at the Society of General Internal Medicine Annual Meeting in Orlando Florida, May 9, 2012.
Conflict of Interest: The authors declare that they do not have a conflict of interest.

Corresponding Author: Stephen D. Persell, MD, MPH; Division of General Internal Medicine and Geriatrics, Feinberg School of Medicine, Northwestern University, Chicago, IL, USA (e-mail: spersell@nmff.org).

\section{REFERENCES}

1. Executive summary of the third report of the National Cholesterol Education Program (NCEP) expert panel on detection, evaluation, and treatment of high blood cholesterol in adults (Adult treatment panel III). JAMA. 2001;285:2486-2497.

2. Grundy SM, Cleeman JI, Merz CN, et al. Implications of recent clinical trials for the National Cholesterol Education Program Adult Treatment Panel III guidelines. Circulation. 2004;110:227-239.

3. Mosca L, Benjamin EJ, Berra $\mathbf{K}$, et al. Effectiveness-based guidelines for the prevention of cardiovascular disease in women-2011 update: a guideline from the American Heart Association. Circulation. 2011;123:1243-1262.

4. Redberg RF, Benjamin EJ, Bittner V, et al. AHA/ACCF [corrected] 2009 performance measures for primary prevention of cardiovascular disease in adults: a report of the American College of Cardiology Foundation/American Heart Association task force on performance measures (writing committee to develop performance measures for primary prevention of cardiovascular disease): developed in collaboration with the American Academy of Family Physicians; American Association of Cardiovascular and Pulmonary Rehabilitation; and Preventive Cardiovascular Nurses Association: endorsed by the American College of Preventive Medicine, American College of Sports Medicine, and Society for Women's Health Research. Circulation. 2009;120:1296-1336.

5. Persell SD, Zei C, Cameron KA, Zielinski M, Lloyd-Jones DM. Potential use of 10-year and lifetime coronary risk information for preventive cardiology prescribing decisions: a primary care physician survey. Arch Intern Med. 2010;170:470-477.

6. Mosca L, Linfante AH, Benjamin EJ, et al. National study of physician awareness and adherence to cardiovascular disease prevention guidelines. Circulation. 2005;111:499-510.

7. Sheridan SL, Crespo E. Does the routine use of global coronary heart disease risk scores translate into clinical benefits or harms? A systematic review of the literature. BMC Health Serv Res. 2008;8:60.

8. Sheridan SL, Viera AJ, Krantz MJ, et al. The effect of giving global coronary risk information to adults: a systematic review. Arch Intern Med. 2010;170:230-239.

9. Hall LM, Jung RT, Leese GP. Controlled trial of effect of documented cardiovascular risk scores on prescribing. BMJ. 2003;326:251-252.

10. Grover SA, Lowensteyn I, Joseph L, et al. Patient knowledge of coronary risk profile improves the effectiveness of dyslipidemia therapy: the CHECK-UP study: a randomized controlled trial. Arch Intern Med. 2007; 167:2296-2303.

11. Edelman D, Oddone EZ, Liebowitz RS, et al. A multidimensional integrative medicine intervention to improve cardiovascular risk. J Gen Intern Med. 2006;21:728-734.

12. Sheridan SL, Shadle J, Simpson RJ Jr, Pignone MP. The impact of a decision aid about heart disease prevention on patients' discussions with their doctor and their plans for prevention: a pilot randomized trial. BMC Health Serv Res. 2006;6:121.

13. Sheridan SL, Draeger LB, Pignone MP, et al. A randomized trial of an intervention to improve use and adherence to effective coronary heart disease prevention strategies. BMC Health Serv Res. 2011;11:331.

14. van Wyk JT, van Wijk MA. Assessment of the possibility to classify patients according to cholesterol guideline screening criteria using routinely recorded electronic patient record data. Stud Health Technol Inform. 2002;93:39-46.

15. Persell SD, Dunne AP, Lloyd-Jones DM, Baker DW. Electronic health record-based cardiac risk assessment and identification of unmet preventive needs. Med Care. 2009;47:418-424.

16. Roland $\mathbf{M}$, Torgerson DJ. What are pragmatic trials? BMJ. 1998;316:285 
17. National Cholesterol Education Program. Risk assessment tool for estimating 10-year risk of developing hard CHD (myocardial infarction and coronary death). Available at http://hin.nhlbi.nih.gov/atpiii/ calculator.asp?usertype=prof. Accessed October 18, 2012.

18. Persell SD, Kaiser D, Dolan NC, et al. Changes in performance after implementation of a multifaceted electronic-health-record-based quality improvement system. Med Care. 2011;49:117-125.

19. Montaño D, Kasprzyk D. Theory of reasoned action, theory of planned behavior, and the integrated behavioral model. In: Glanz K, Rimer BK, Viswanath $\mathbf{K}$, eds. Health Behavior and Health Education: Theory, Research, and Practice. 4th ed. San Francisco: Wiley; 2008:67-96.

20. D'Agostino RB Sr, Vasan RS, Pencina MJ, et al. General cardiovascular risk profile for use in primary care: the Framingham heart study. Circulation. 2008;117:743-753.

21. Wood DA, Kotseva K, Connolly S, et al. Nurse-coordinated multidisciplinary, family-based cardiovascular disease prevention programme (EUROACTION) for patients with coronary heart disease and asymptomatic individuals at high risk of cardiovascular disease: a paired, cluster-randomised controlled trial. Lancet. 2008;371:19992012.

22. Wister A, Loewen N, Kennedy-Symonds H, McGowan B, McCoy B, Singer $\mathbf{J}$. One-year follow-up of a therapeutic lifestyle intervention targeting cardiovascular disease risk. CMAJ. 2007; 177:859-865.

23. Randomised controlled trial evaluating cardiovascular screening and intervention in general practice: principal results of British family heart study. Family Heart Study Group. BMJ. 1994;308:313-320.

24. Benner JS, Erhardt L, Flammer M, et al. A novel programme to evaluate and communicate 10-year risk of $\mathrm{CHD}$ reduces predicted risk and improves patients' modifiable risk factor profile. Int $\mathrm{J}$ Clin Pract. 2008;62:1484-1498.

25. Mann DM, Ponieman D, Montori VM, Arciniega J, McGinn T. The Statin choice decision aid in primary care: a randomized trial. Patient Educ Couns. 2010;80:138-140.

26. van Wyk JT, van Wijk MA, Sturkenboom MC, Mosseveld M, Moorman PW, van der Lei J. Electronic alerts versus on-demand decision support to improve dyslipidemia treatment: a cluster randomized controlled trial. Circulation. 2008;117:371-378. 